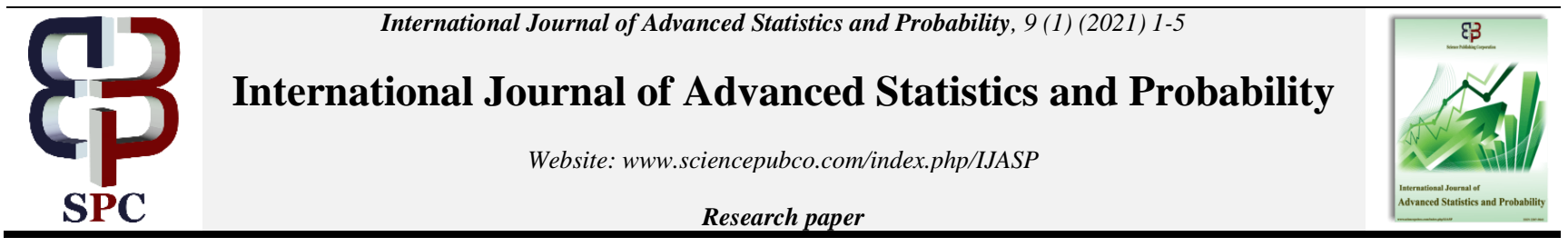

\title{
Solving practical waste collection with time windows in an urban area
}

\author{
Abdulwahab Almutairi * \\ School of Mathematics, Unaizah College of Sciences and arts, Qassim University, Saudi Arabia \\ *Corresponding author E-mail: a.abdulwahab.a@gmail.com
}

\begin{abstract}
In logistics, several algorithms can be implemented in order to solve the problems of the vehicle routing with variants in order to find near-optimal solutions. Waste Collection can be considered as an essential logistic activity within any area or city. This kind of paper is aimed to implement Iterated greedy (IG) and Adaptive Large Neighborhood Search (ALNS) to solve waste collection vehicle routing problem with time windows on a real-case study. The idea is to generate an efficient way to collect waste problems in an area located in Riyadh, Saudi Arabia. Moreover, generating a route plays a significant role in terms of serving all customers' demands who have own different time windows of receiving goods. Also, the performance of the proposed algorithms according to all instances is examined and minimizing the total costs and meeting all constraints that related to capacity, time windows, and others. To evaluate the execution of the presented algorithms, the computational results showed essential improvements, and also ALNS algorithm generates reasonable solutions in terms of total costs and a reasonable amount of time, when compared to other algorithms.
\end{abstract}

Keywords: ALNS; Iterated Greedy; Time Window; Waste Collection.

\section{Introduction}

The Vehicle Routing Problem (VRP) has been considered as one of the known combinatorial optimization problems in the class of NPhard (8). The classical model of VRP can be classified as a problem related to generating an optimal collection or delivery routes from either one or many central depots to a set of known customers' locations or collection node(s) with specific constraints. Also, the VRP is aimed at identifying a number of routes for a set of vehicles from a central depot to a number of customers in order to serve them and traveling back to the central depot, without exceeding the capacity of the vehicle constraints of the vehicles at a minimum total cost. Many previous studies reported for both theoretical and practical purposes. For example, this paper aims to solve one of the waste management problem that related to the VRP, mainly focusing on real life case study of pick-up waste collection problem. The problem can be summarized as follows: the waste collection problem contains several routing vehicles in order to collect/pick-up customers' waste within a specific time and try to minimize the total travel cost or maximizing profits. This kind of problem can be called as the Waste Collection Vehicle Routing Problem with Time Windows (WCVRPTW). WCVRPTW differs from the traditional VRPTW by that the waste-collecting vehicles have to empty their load at the central depot. WCVRPTW differs from the traditional VRPTW by that the vehicles have to be empty the vehicle when traveling back to the central depot. Note that many trips to the central depot can be allowed for each vehicle.

The waste collection cost is typically measured in terms of cost per ton, with an inverse relationship between the waste collection costs and the amount of materials collected. Therefore, moving waste bins that are only partially full could be an unnecessary misuse of resources, and an avoidable production of polluting emissions. The waste collection problem is divided into three major areas: residential, commercial, and roll-on-roll-off. Each area includes a specific waste and re-cycling material also each area is very different from others. Residential waste collection generally involves visiting private homes; Residential waste has different categories of service, e.g., recycling, yard waste and garbage. Commercial waste collection involves visiting customers with large containers such as restaurants, small office buildings, and malls. Typically, each category needs at least a one-day-per-week service. For vehicles and drivers, drivers have to limit each day by the amount of time on a weekday. At the same time, the vehicles have to travel back to the central depot when the vehicles are full. Each vehicle can make several travels per day to serve the customers. For the most part, routes and schedules are known. The difference between roll-on- roll-off collection area and commercial collection area is the container size such as using large containers at construction sites or other high-volume locations. Note that each vehicle can carry a single trailer at a time. Both vehicles and drivers are limited by the number of hours in a workday. This paper focuses on a specific kind of residential waste collection in which waste is located in bins (customer location) along the streets of a defined road network. The structure of the present article is organized as follows: Section 2 offers a description of the dataset of the pick-up case study on waste collection problem. The literature review for VRPTW is shown in section 3. Section 4 proposes the IG algorithm and the ALNS algorithm. Computational experimentation is discussed in Section 5. Finally, our conclusion with future work is shown in section 6. 


\section{Problem description}

The waste collection problem can be formulated as WCVRPTW with various requirements could exist in real case situation. A variety of real-case studies have previously been presented with different methodologies in the literature. In this section, the work is carried out in the biggest city of Saudi Arabia (Riyadh) and a sample dataset of the waste collection/pick-up problem is selected. Customers' locations: There are nine areas and involved one central depot. There are significant constraints during the design of the route vehicle for waste collection problem. Each area has number of customers' locations at different geographical locations with different demands. Vehicle capacity and number of vehicles: There is a strict restriction on large vehicles during serving the customers' demands at daytime in urban areas, hence the small vehicles capacities are chosen because they can run at any time. The vehicle has to travel back when it's a full capacity to empty it. A vehicle has to travel and return back to the central depot. Time windows: each customer has own time windows (i.e. earliest and latest service) and will accept to serve in own time range. While minimizing the total costs is one of the main objectives of this real-case study, the time windows also have to be considered. This can be called as the vehicle routing problem with time windows that is usually dealt with the mathematical procedures. Clarke and Wright saving algorithm (initial solution) with other algorithms have been used to generate route network and find the near-optimal solution.

\section{Literature review}

Improving the efficiency of transport the customers' demands from the central depot is highly linked to the subject called the VRP. The VRP is considered as an important combinatorial optimization. VRP is introduced (9) based on a case study called a real gasoline delivery problem. Also, the VRP can be considered as a significant area in the operational research. Briefly, the VRP deals with transport the demands between customers' locations and central depots. All vehicles have to begin and travel back to the central depot. Each customer location has to be served once during the trip. The main aim of the VRP is to generate a minimum number of routes that serve all customers' demand. Note that in the theoretical side, the VRP is simple, easy to explain and understand. However, it can be hard to apply and solve in a specific real-life scenario, especially when the decision-makers used additional practical constraints. The VRP can be linked with other variants such as heterogeneous VRP, periodic vehicle routing problem (PVRP) and VRP with Time Windows (VRPTW). This research focuses on VRPTW mainly.

In addition to the number of vehicles and capacity constraints, time window constraints arise in many case studies related to VRP and it is difficult to obtain a feasible solution. The Capacitated Vehicle Routing Problem with Time Windows is considered as the CVRP with Time Window constraints. In a real case study, a set of customers' locations must only be visited in a specific period time; therefore, we refer to this kind of period time as the time windows (soft/hard). Hence, time window of serving the customers would be considered. In CVRPTW, the demands of customers have to start to be served within a time window period [Ei, Li]. If a driver of a vehicle arrives at customer location i before $\mathrm{Ei}$, vehicles have to wait until $\mathrm{Ei}$, since there is no extra cost for waiting. If the driver of the vehicles arrives at the location of the customer $\mathrm{i}$ after $\mathrm{Li}$, as the customer location is not available, the vehicle driver cannot serve and pick up anything. An additional penalty costs have to be considered.

(13) present a real life-case study of waste collection vehicle routing problem with time windows with several considerations, such as multiple trips and drivers' lunch breaks. In order to find a solution in this real-life problem, they have been successfully implemented and deployed algorithms for the practical real-life waste collection problems of waste management with a simplified mathematical model. The case study has been applied in North America with nearly 26,000 collection and transfer vehicles. An adaptive large neighborhood search algorithm has been proposed (6) to solve a real case study that provided by a number of instances from two sources namely a literature and a Danish garbage collection company. The paper aims to generate an efficient way with optimal costs for garbage bins and to solve the waste collection vehicle routing problem with time windows. Authors concluded that the proposed algorithm presents good solutions for using benchmark instances.

(18) proposed a heuristic and route network in order to minimize the total costs travelled for VRPTW. They start the solution with the classical saving algorithm along with adding time window and capacity restraints at the each customer location. In terms of the results, the results of the case study of ICD TIEN SON (VIETNAM)N is better because it serves and carries all freight at the same time with a small number of vehicle drivers and a smaller number of travelled kilometers during the route. In (15) an integer linear programming algorithm was proposed in order to minimize the total travel time of the real case study. The result was achieved through three phases of defining the starting points and clustering set covering. The second phase is assigning busses from each starting point; the final phase is solving the SDVRP with time windows through determining the route. These three phases can be done by using an exact algorithm. The experiment of the time was relatively lengthy, the experiment results showed that the generated result was more suitable than the both current routing and scheduling that the firm used. In the end, the result was also able to reduce fuel costs by $9 \%$ that was achieved from a shorter total travel distance by the shuttle buses.

(7) proposed two solution approaches the hybrid particle swarm optimization (HPSO) with simulated annealing, and the other being the conventional particle swarm optimization (PSO) in order to solve a multi-compartment vehicle routing problem with time window (MCVRPTW). They concluded their computational results with that both approaches generated an efficient solution for the MCVRPTW. However, the HPSO algorithm produced better solutions than the PSO algorithm especially in delivering the best solution. (23) A hybrid particle swarm optimization algorithm and a hybrid genetic algorithm has been presented in order to solve a practical problem which is related to a multi-depot, multi-trip VRPTW and release dates. The aim behind using this kind of method is to generate a set of trips for the number of vehicles supplied by different central depots in order to minimize total traveling time. They tried to focus on customer's time windows and the release date of customers' packages. From the computational results side, a mixed-integer programming model with the algorithms can generate near solutions for both small-scale and large-scale instances in reasonable computation time. (6) They took into consideration the times and demand with a mathematical model for solving the time-dependent vehicle routing problem with time windows (TDVRPTW) in order to minimize the total costs. An improved ant colony algorithm with a congestion avoiding approach used to show a best way to reduce the total travel costs, departure time and driving route for each vehicle.

\section{Methodology}

The VRPTW is a hard problem to solve and it has been done by (Katja and Allan). In this study, we propose an effective heuristic for minimizing the total travel costs in a real case study. Three steps have been described in details in order to find near optimal solution of 
the real-life case study problem in the following subsections. Therefore, the first step is based on a well-known algorithm CWS, which was proposed in (23) to generate a feasible initial solution for VRPTW. In the second step, we applied the ALNS algorithm and IG in the solution obtained in the first step in a parallel way. In the final step, the solutions have been re-optimized by using the local search, which has been added in order to improve the final solution for each route.

\subsection{Clarke and wright saving algorithm}

The CWS algorithm is considered one of the most popular heuristics for the classical vehicle routing problem. This method was developed by Clarke and Wright in 1964. The CWS heuristic can work in a parallel or sequential way. The first step is that it starts with a solution in which each customer's location is serviced individually by a separate route (travel between central depot and customer and come back to central depot). Then next combining the two routes serving, customers $i$ and $\bar{j}$, respectively, results in a cost savings of $S_{i j}=d_{i 0}+d_{0 j}-d_{i j}$ and combing the two routes has to be feasible. Also, during the combination, the total demand has to be not exceeding the capacity of the vehicle. The CWS heuristic is illustrated in Figure 1.
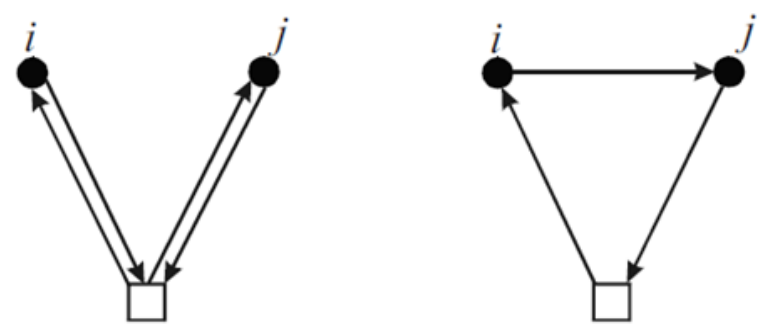

Fig. 1: The Saving Heuristic.

\subsection{ALNS algorithm}

The ALNS algorithm is an extension of other algorithm called large neighborhood search. The framework of the ALNS algorithm has proposed by (21). The idea behind the ALNS is to repeatedly remove customers from the route solution and then reinsert them at a more profitable location. This situation is defined implicitly through named both destroy operators and repair operators therefore the ALNS algorithm applies both destroy and repair operators in each iteration. The processes of both removal and insertion operators are adapted from (21). To move from one/current solution to other solution, The ALNS first destroys part of the solution and then repairs the partial solution to restore a full solution, which most likely will be different from the beginning point. For a VRP, the destroy operator is to remove some customers' demands from the feasible solution and then allow the repair operator reintroduce removed customers' demands into the solution. The new solution will be taken into account when the new solution is better than the current solution.

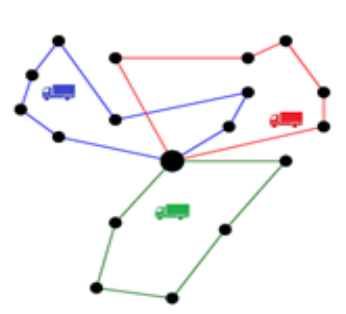

Fig. 2: Initial Solution

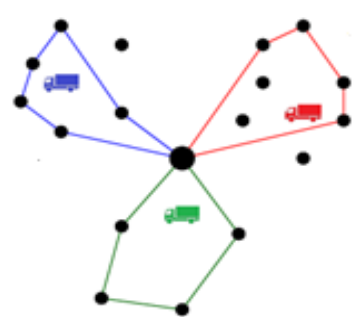

Fig. 3: Destroy Operator.

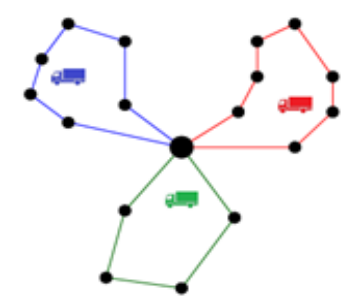

Fig. 4: Repair Operator.

This is illustrated in Figure 2.4. In Figure 2, the current solution with three routes are shown, in Figure 3, the destroy operation is presented by removing four customers, in Figure 4, we present the solution after reinserted the four customers.

\subsection{IG algorithm}

This algorithm has been applied in traditional permutation flow shop scheduling problems (19 and 20) and then has been developed by ( 2 and 3 ) in order to find a solution to the vehicle routing problem. The steps of the IG algorithm are destruction, construction, optimization and finally acceptance criteria. In the destruction step, some solution components are selected and removed from a previous solution. In the construction step, after removing components from the destruction phase, construction procedure is applied by re-assigned to different positions to minimize the cost, and local search includes in order to improve each solution. The acceptance criteria are to compare the new solution with the current solution in order to select the better solution with no constraint violation.

\subsection{Local search improvement}

In general, local search (LS) algorithms have been applied in solving numerous computational optimization problems, such as the VRP. A local search can be defined as a classical optimization method that able to generate an optimal local solution to the problem. The aim of using the local search is to move from one solution to a better solution. Therefore, local search for route planning can be improved within a single route or between routes. In this study, we try to focus on locally improve the same route by using three local searches which are 1-0 exchange, 1-1 exchange, 2-opt exchange and achieve a good solution. The process of each local search is repeated until no further improvement can be found, and then this solution is called a locally optimal solution. The design of these local searches structure is defined as follows: 
The first local search is 1-0 exchange. The 1-0 exchange can be done in order to improve the current solution by removing a customer from one location and insert it in the good location in the same route.

The second local search is 1-1exchange. The 1-1 exchange can be achieved by selecting two customers on the same route and then insert them in different customers' locations.

The final local search is 2-opt heuristic. The 2-opt heuristic aimed to improve a single-vehicle route exchanges two pairs of arcs in the same route in order to reduce and improve the travel total cost of the route solution.

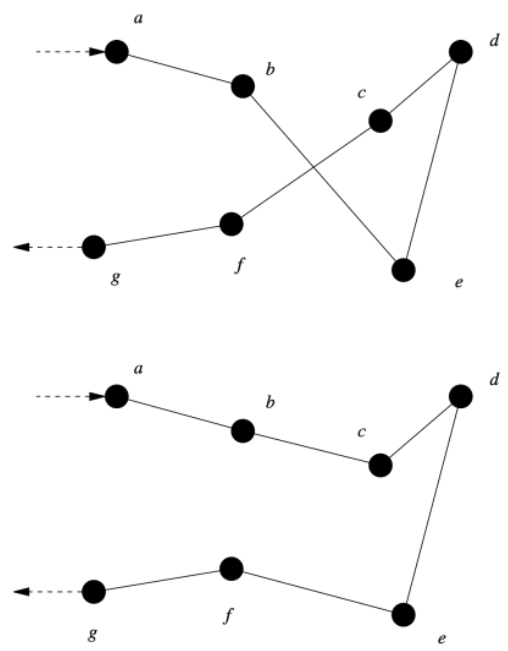

Fig. 5: 2-opt Heuristic.

\section{Computational results}

We experiment with instances from the practical real-life pick-up problem in Riyadh. Each instance has one depot and several numbers of customers. Also, all the vehicle capacity is taken from the company. The table has nine instances and each one links with a specific area. All the computational results are shown in Table 1 were coded in JAVA on a Macintosh HD on 2.3 GHz Intel HD Graphics 4000 $1024 \mathrm{MB}$ with Intel Core i7 with 4 GB $1600 \mathrm{MHz}$ DDR3 in order to find an optimal solution of the instances. Table 1 displays several columns. The first column represents the instances e.g. 3296_2_173, which 3296 represents the number of customers, 2 represents the number of the vehicle that used and 173 represents the total mile distances of the route. The second column represents the number of vehicle capacity used. The third column shows the number of vehicle stops. The computational results obtained for the real-life pick-up problem by applying IG and ALNS are shown in between 4 to 7 columns. The solution costs are presented in columns (4) and (6), while the solution time (in second) is presented in column (5) and (7). The last two columns represented the improvement between these algorithms. Based on the computational results presented in the table, it can be concluded that the solution from the ALNS method achieved better solution in terms of both solutions "total costs travelled and total travel time", in comparison to IG algorithms solutions. Furthermore, the percentage deviation formula can be calculated between the proposed algorithms as follows;

Percentage deviation $=\left(\frac{(\text { old solution })-(\text { new solution })}{(\text { new solution })}\right) * 100$.

From table 1, it is possible to see the summary of the results achieved by IG and ALNS where the capacity of the vehicle is $100 \%$ during the design stage. Note that the computational time reported in second and from the average of all the instances presented at the last row of Table 1, it is evident that the ALNS algorithm should be preferred, having a lower average both total travel costs and total travel times than the IG algorithm. Generally, the results generated in Table 1 indicate that the results are improved in all of the instances. The gaps can be expressed as a percentage improvement value when compared between two algorithms and also the respective gaps between total travel costs and total times are presented in Table 1 under the column (8) and the column (9). The improvement is mostly quite small and cannot be reached 1\% on both costs and times. Also, the 955_1_43.33 and 3342_1_176 instances have the lowest gap over all experiments in terms of total costs and total time respectively. Similarly, the 6498_3_351.33 and 3281_1_172.5 have the most significant gap in all the experiments in terms of the total costs and total time. According to the gap column, it is clear that the ALNS algorithm presented in this paper always obtains better results. Consequently, the solutions clearly indicated that using the ALNS algorithm in those instances helps to obtain good solutions in both a reasonable amount of time and total costs and on average better results than the IG algorithm. Furthermore, the better solution for each instance in the table is presented in bold. ALNS outperforms the IG algorithm for both total costs and times.

Table 1: Summary of Computational Results IG and Adaptive Large Neighborhood Search

\begin{tabular}{|c|c|c|c|c|c|c|c|c|}
\hline Instance & $\begin{array}{l}\text { \# of used } \\
\text { capacity }\end{array}$ & \# of stops & $\begin{array}{l}\text { Total costs } \\
\text { (IG) }\end{array}$ & $\begin{array}{l}\text { TT (sec) } \\
\text { (IG) }\end{array}$ & $\begin{array}{l}\text { Total costs } \\
\text { (ALNS) }\end{array}$ & $\begin{array}{l}\text { TT (sec) } \\
\text { (ALNS) }\end{array}$ & $\begin{array}{l}\text { Improvement } \\
\text { cost IG-ALNS }\end{array}$ & $\begin{array}{l}\text { Improvement } \\
\text { time IG-ALNS }\end{array}$ \\
\hline 3296_2_173 & $100 \%$ & 3296 & 364.9 & 15 & 362.1 & 13 & $0.77 \%$ & 0.15 \\
\hline 2973_1_155.5 & $100 \%$ & 2973 & 450.5 & 9 & 448.1 & 8 & $0.53 \%$ & 0.12 \\
\hline 3264_1_171.66 & $100 \%$ & 3264 & 315.6 & 13 & 313.2 & 11 & $0.76 \%$ & 0.18 \\
\hline 3147_1_165.16 & $100 \%$ & 3147 & 537.6 & 11 & 535.2 & 8 & $0.44 \%$ & 0.37 \\
\hline 6498_3_351.33 & $100 \%$ & 6498 & 567.3 & 30 & 562.5 & 22 & $0.85 \%$ & 0.36 \\
\hline 3297_1_173.5 & $100 \%$ & 3297 & 334.5 & 16 & 332.9 & 13 & $0.48 \%$ & 0.23 \\
\hline 3281_1_172.5 & $100 \%$ & 3281 & 354.4 & 14 & 352.8 & 10 & $0.45 \%$ & 0.40 \\
\hline 3342_1_176 & $100 \%$ & 3342 & 345.6 & 15 & 343.2 & 14 & $0.69 \%$ & 0.07 \\
\hline 955_- $1 \_\overline{4} 3.33$ & $100 \%$ & 955 & 433.6 & 4 & 432.5 & 3 & $0.25 \%$ & 0.33 \\
\hline Average & $100 \%$ & 3339.2 & 411.55 & 14.11 & 409.16 & 11.33 & $0.58 \%$ & 0.33 \\
\hline
\end{tabular}




\section{Conclusion}

In this study, we focused on a real-life case study of a waste collection formulated as vrpst. The solution of the problem was found through two algorithms which are ig and alns in order to pick-up waste collection problems by considering the constraints of time windows in riyadh city. In the first stage, initial feasible solutions have been generated and then the solutions improved by implemented an ig and anls in the second stage. The instances used consisted of nine areas with one central depot and coded in a software system for solving the problem. The computational experiments can be presented that the prosed algorithm, alns achieved better solution than other algorithms in terms of total costs and time windows. In future work, time-dependency is one of the crucial constraints and it can consider time-dependent travel times in the problem since speeds vary throughout the day due to the events like congestion or accidents during the rush hours. Therefore, we must have two parts which are stochastic and dynamic travel times. The structure in the algorithm has to adjust with respect to the distributions of the arrival times.

\section{References}

[1] Almutairi A. (2020). Combined Nearest Greedy algorithm with randomised algorithm to solve waste collection problem. International Journal of Statistics and probability. Vol.9,No.3.DOI:10.5539/ijsp.v9n3p66. http://www.ccsenet.org/journal/index.php/ijsp/article/view/0/42581. https://doi.org/10.5539/ijsp.v9n3p66.

[2] Almutairi A. (2018). Solving capacitated vehicle routing problem by iterated greedy algorithm. Journal of Natural Science and Maths. JNM:2018 MATH-02

[3] Almutairi A. (2018). Using randomization into iterated greedy algorithm in order to solve capacitated vehicle routing problem. Journal of Natural Science and Maths. JNM: 2018 MATH-03.

[4] Braaten, S., Gjonnes, O., Hvattum, L, M., tirade, G., (2017). Heuristic for the robust vehicle routing problem with time windows. Expert system with applications. 77(2017) 136-147. https://doi.org/10.1016/j.eswa.2017.01.038.

[5] Buhrkala, K., Larsena, A., Ropkea, S., (2012). The waste collection vehicle routing problem with time windows in a city logistics context. The Seventh International Conference on City Logistics. Procedia - Social and Behavioral Sciences 39 (2012) 241 - 254. https://doi.org/10.1016/j.sbspro.2012.03.105.

[6] Changshi Liu, C. Kou, G., Zhou, X., Peng, Y., Sheng, H., Alsaadi, F. 2019. Time-dependent vehicle routing problem with time windows of city logistics with a congestion avoidance approach. Knowledge-Based Systems.

[7] Chena. J., Shic, J., 2019. A multi-compartment vehicle routing problem with time windows for urban distribution - A comparison study on particle swarm optimization algorithms. Computers \& Industrial Engineering 133 (2019) 95-106. https://doi.org/10.1016/j.cie.2019.05.008.

[8] Demir, E., Bektas, T., Laporte, G., (2012). An adaptive large neighbourhood search heuristic for the pollution-routing problem. European Journal of operational research 223(2012)364-359. https://doi.org/10.1016/j.ejor.2012.06.044.

[9] Dantzig, G, B., Ramser, J, H. (1959). The truck dispatching problem, management science, Vol. 6, No. 1, pp. 80-91. https://doi.org/10.1287/mnsc.6.1.80.

[10] El-sherbeny, N., (2010). Vehicle routing with time windows: an overview of exact, heuristic and metaheuristic methods. Journal of king Saud University (science). 2010. 22, 123-131. https://doi.org/10.1016/j.jksus.2010.03.002.

[11] Faccio, M., Persona, A., Zanin. G., (2011). Waste collection multi objective model with real time traceability data. Waste management. 31 (2011) 2391-2405. https://doi.org/10.1016/j.wasman.2011.07.005.

[12] Gu, W., Cattaruzza, D., Ogier, M., Semet, F., adaptive large neighbourhood search for the commodity constrained split delivery VRP. Computer and operations research 112(2019) 104761. https://doi.org/10.1016/j.cor.2019.07.019.

[13] Kim, B., Kim, S., Sahoob, S. (2006). Waste collection vehicle routing problem with time windows. Computers \& Operations Research 33 (2006) 3624-3642. https://doi.org/10.1016/j.cor.2005.02.045.

[14] Liu, X., Laporte, G., Chen, Y., He, R., (2017). An adaptive large neighbourhood search metaheuristic for agile satellite scheduling with timedependent transition time. Computer and operations research. S0305-0548(17)30097-7. https://doi.org/10.1016/j.cor.2017.04.006.

[15] Latiffianti, E., Siswanto, N., Firmandani, R, A. (2017). Split delivery vehicle routing problem with time windows: a case study. International conference on industrial and system engineering (IConISE). https://doi.org/10.1088/1757-899X/337/1/012012.

[16] Miranda, D, M., Conceicao, S, V., (2016). The vehicle routing problem with hard time windows and stochastic travel and service time. Expert system with application 64 (2016) 104-116. https://doi.org/10.1016/j.eswa.2016.07.022.

[17] Mattos Ribeiro, G., Laportea, G., (2012). An adaptive large neighborhood search heuristic for the cumulative capacitated vehicle routing problem. Computer \& operations research. 39 (3) 728-735. https://doi.org/10.1016/j.cor.2011.05.005.

[18] Nghia, N, H., Hieu, N, M., Linh, N, T, T., (2016). Solving practical vehicle routing problem with time windows (VRPTW)- a case study of ICD time son (Vietnam).

[19] Ruiz R, Stutzle T. An Iterated Greedy Algorithm for the Flowshop Problem with Sequence Dependent Setup Times. The 6th Metaheuristics International Conference, 2005. 817-823.

[20] Ruiz R, Stutzle T. A simple and effective iterated greedy algorithm for the permutation flowshop scheduling problem. Euro Journal of Opera Res, 2007. 177, 2033-2049. https://doi.org/10.1016/j.ejor.2005.12.009.

[21] Ropke, S., Pisinger, D., 2006. An adaptive large neighbourhood search heuristic for the pickup and delivery problem with time windows. Transportation Science. 40(4), 455-472. https://doi.org/10.1287/trsc.1050.0135.

[22] Tas, D., Dellaert, N., Woensel, T., De Kok, T., (2013). Vehicle routing problem with stochastic travel times including soft time windows and service costs. Computer \& operations research 40 (2013) 214-224. https://doi.org/10.1016/j.cor.2012.06.008.

[23] Zhena, L., Maa, C., Wanga, K, Xiaoa, L., Zhangc, W., (2020). Multi-depot multi-trip vehicle routing problem with time windows and release dates. Transportation Research Part E 135 (2020) 101866. https://doi.org/10.1016/j.tre.2020.101866. 